\title{
REGULATION OF PYRUVATE METABOLISM BY THE MITOCHONDRIAL ENERGY STATE: THE EFFECT OF PALMITYL-COENZYME A
}

\author{
M. LOPES-CARDOZO, W.J. VAARTJES and S.G. VAN DEN BERGH \\ Laboratory of Veterinary Biochemistry, State University of Utrecht, Biltstraat 172, Utrecht, The Netherlands
}

Received 26 October 1972

\section{Introduction}

In isolated rat liver mitochondria pyruvate can either be decarboxylated or carboxylated. In the former case the pyruvate dehydrogenase complex (EC 1.2.4.1) oxidizes pyruvate to acetyl-CoA ( $\mathrm{AcCoA})$ leading to citrate synthesis or ketone-body formation. In the latter case pyruvate is converted to oxaloace tate by the action of pyruvate carboxylase (EC 6.4.1.1). Oxaloacetate may enter the gluconeogenic pathway by its conversion into phosphoenolpyruvate. Alternatively, it may depress ketogenesis by diverting $\mathrm{AcCoA}$ towards the synthesis of citrate which is considered to be a lipogenic precursor [1].

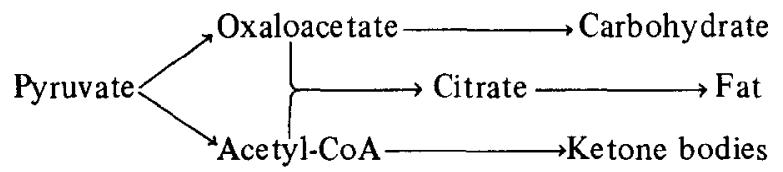

From the scheme it is evident that the control of mitochondrial pyruvate metabolism is of key importance in the regulation of gluconeogenesis, ketogenesis and lipogenesis in liver [2,3].

Several factors have been reported to modify the activities of isolated pyruvate dehydrogenase [4-7] and pyruvate carboxylase [8-11]. Increasing $\mathrm{AcCoA} /$ $\mathrm{CoA}, \mathrm{ATP} / \mathrm{ADP}$ and $\mathrm{NADH} / \mathrm{NAD}^{+}$ratios may cooperatively switch mitochondrial pyruvate metabolism from decarboxylation to carboxylation [12]. This regulatory pattern has been confirmed in isolated liver mitochondria [13-17].
Recently the in teresting suggestion was brought forward that pyruvate metabolism is affected indirectly by the level of long-chain acyl-CoA esters. Longchain acyl-CoA esters like palmityl-CoA strongly inhibit adenine nucleotide (AdN) translocation through the inner mitochondrial membrane [18-23]. A reduced rate of $A d N$ transport would enhance the intramitochondrial ATP/ADP ratio since the maximal rates of AdN transport and ATP synthesis are of the same order of magnitude [24]. As mentioned above, the ATP/ADP ratio is one of the factors controlling the activity of pyruvate carboxylase $[16,17]$. Consequently, the level of acyl-CoA esters may be of importance in the control of pyruvate carboxylation in vivo. This effect of acylCoA esters would be comparable to their stimulatory effect on ke togenesis in liver mitochondria described earlier [25].

In the present report the effect of State-3 to State-4 transitions on pyruvate metabolism is compared with the effect of palmityl-CoA. Our results are compatible with the conclusion of Walter and coworkers [16, 17] that, at optimal substrate concentrations, pyruvate carboxylation is mainly regulated by the phosphorylation state of the AdN. At elevated ATP/ADP ratios pyruvate oxidation is diminished whereas pyruvate carboxylation is stimulated leading to an accumulation of Krebs-cycle intermediates and a lowered ketonebody formation from pyruvate. A similar stimulation of pyruvate carboxylation can be brought about by palmityl-CoA, presumably due to an inhibition of $\mathrm{AdN}$ translocation and a concomitant increase in the mitochondrial ATP/ADP ratio.

* To whom to address correspondence. 


\section{Methods and materials}

Oxygen uptake was measured at $25^{\circ}$ with a Gilson Respirometer. The standard reaction medium contained $50 \mathrm{mM}$ sucrose, $5 \mathrm{mM} \mathrm{MgCl}, 2 \mathrm{mM}$ EDTA, $15 \mathrm{mM}$ $\mathrm{KCl}, 50 \mathrm{mM}$ Tris- $\mathrm{HCl}, 30 \mathrm{mM}$ potassium phosphate, $30 \mathrm{mM}$ glucose, $0.5 \mathrm{mM} \mathrm{ADP}$ and $25 \mathrm{mM} \mathrm{KHCO}_{3}$, $\mathrm{pH}$ 7.5. Reactions were started by the addition of the mitochondria. Final volume, $2.0 \mathrm{ml}$. After $16 \mathrm{~min} 1$ $\mathrm{ml}$ ice-cold $\mathrm{HClO}_{4}(1.2 \mathrm{~N})$ was added and me tabolites were assayed spectrophotometrically in the deproteinized supernatants using standard enzymic methods.

Pyruvate carboxylation was measured as the increase in malate, fumarate and citrate. It has been shown [26] that over $90 \%$ of the pyruvate carboxylated is recovered in these intermediates. Addition of fluorocitrate to block the oxidation of citrate proved to be less satisfactory. In our hands $200 \mu \mathrm{M}$ potassium fluorocitrate was needed to inhibit completely the oxidation of added citrate. At this concentration of fluorocitrate, however, a $50 \%$ inhibition of pyruvate carboxylation was observed.

The relative rate of ketogenesis is expresse $d$ as the acetyl-ratio [27], i.e. the quotient of AcCoA converted into ketone bodies and the total flux through the AcCoA pool:

acetyl-ratio $=\frac{2 \times \text { ketone bodies formed }}{\text { pyruvate used }- \text { pyruvate carboxylated }}$

The 3-hydroxybutyrate/ace toace tate (HB/Acac) ratio is used as an indicator of the mitochondrial NADH/ $\mathrm{NAD}^{+}$ratio [28].

L-Palmitylcarnitine was synthesized according to Bremer [29]. L-Carnitine chloride was a generous gift of Otsuka Pharm. Fact. (Osaka). Palmityl-CoA was obtained from Serva; barium fluorocitrate and carbonyl-cyanide- $m$-chlorophenylhydrazone (CCCP) from Calbiochem. Other biochemicals and experimental details were exactly as previously described $[20,25]$.

\section{Results and discussion}

\subsection{Influence of the mitochondrial energy state on pynuvate metabolism}

As can be seen from table 1, under State- 4 conditions (hexokinase absent) in the presence of $25 \mathrm{mM} \mathrm{KHCO}_{3}$ 266
Table 1

Effects of hexokinase and L-malate on pyruvate metabolism.

\begin{tabular}{|c|c|c|c|c|c|}
\hline \multirow{3}{*}{ Additions } & \multicolumn{4}{|c|}{ Pyruvate } & \multirow{3}{*}{$\frac{\mathrm{HB}}{\mathrm{Acac}}$} \\
\hline & Used & $\begin{array}{l}\text { Carbox- } \\
\text { ylated }\end{array}$ & $\begin{array}{l}\text { Converted } \\
\text { into ke tone } \\
\text { bodies }\end{array}$ & \multirow{2}{*}{$\begin{array}{l}\text { Carbox- } \\
\text { ylated } \\
\text { Decarbox- } \\
\text { ylated }\end{array}$} & \\
\hline & \multicolumn{3}{|c|}{ ( $\mu$ moles) } & & \\
\hline None & 4.84 & 2.37 & 0.44 & 0.96 & 0.47 \\
\hline Hexokinase & 3.67 & 0.54 & 1.18 & 0.17 & 0.16 \\
\hline Nonet & 2.15 & 0.40 & 1.22 & 0.23 & 0.65 \\
\hline Hexokinase ${ }^{\dagger}$ & 2.62 & 0.08 & 1.40 & 0.03 & 0.13 \\
\hline L-Malate & 4.22 & 1.58 & 0.26 & 0.60 & 0.44 \\
\hline
\end{tabular}

$\dagger$ In these experiments $\mathrm{KHCO}_{3}$ was replaced by an equimolar concentration of $\mathrm{KCl}$.

The standard reaction medium (see Methods) was supplemented with $3 \mathrm{mM}$ pyruvate. Further additions: 2.6 units of hexokinase, $1 \mathrm{mM} \mathrm{L}$-malatc. Mitochondrial protein, $11.0 \mathrm{mg}$.

about half of the pyruvate metabolized is carboxylated and less than $10 \%$ is converted in to ketone bodies. Under State- 3 conditions (hexokinase added) with added bicarbonate pyruvate carboxylation is markedly depressed and parallel increases in pyruvate oxidation and in ketone-body formation are observed. Virtually no pyruvate carboxylation occurs in a State-3 medium without $\mathrm{KIICO}_{3}$. It is noteworthy that added malate also inhibits pyruvate carboxylation. This inhibition may be explained either by a direct effect of malate (or oxaloace tate) on pyruvate carboxylase or by an efficient removal of $\mathrm{AcCoA}$ which is an activator of pyruvate carboxylase [8-11].

In fig. 1 the time courses of pyruvate carboxylation and of ketogenesis are compared in various energy states. Obviously, the acetyl-ratio (see Methods) is inversely related to the rate of pyruvate carboxylation. In uncoupled mitochondria the accumulation of Krebs-cycle intermediates is negligible and the acetylratio increases to about 0.75 . On the other hand, under State- 4 conditions pyruvate carboxylation is an active process and the acetyl-ratio decreases from 0.30 to about 0.15 .

The findings of table 1 and fig. 1 support the conclusion of Walter and coworkers $[16,17]$ that under these in vitro conditions the rate of pyruvate carboxylation is mainly determined by the phosphorylation 


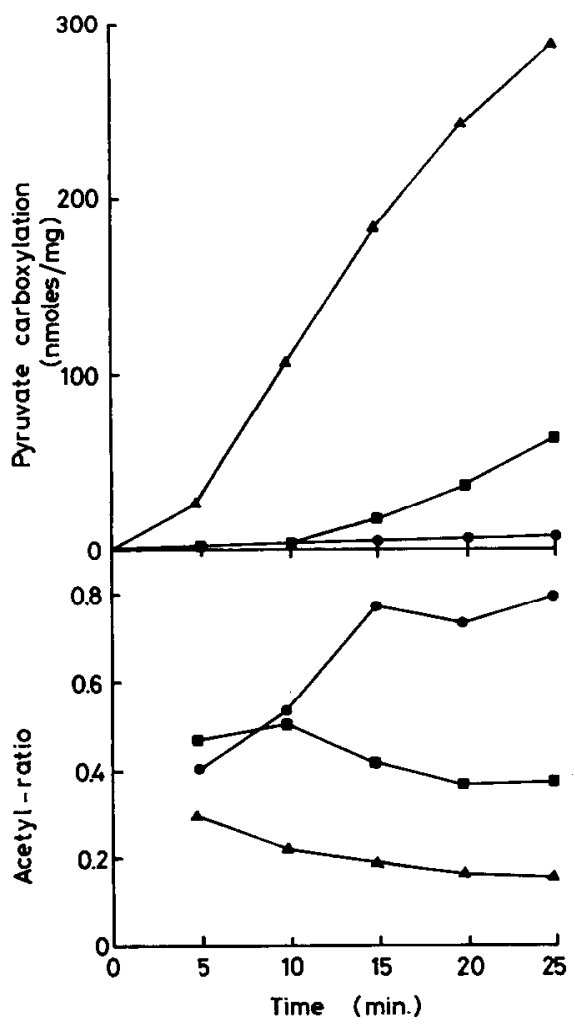

Fig. 1. Time course of pyruvate carboxylation and ketogenesis in various energy states. The standard reaction medium was supplemented with $5 \mathrm{mM}$ pyruvate. Further additions as indicated below. After addition of the mitochondria $15.0 \mathrm{ml}$ incubations were shaken vigorously in open Erlenmeyer flasks at $25^{\circ}$. At the times indicated $2.0 \mathrm{ml}$ aliquots were withdrawn and analyzed. Mitoch ondrial protein, $4-6 \mathrm{mg} / \mathrm{ml}$ reaction

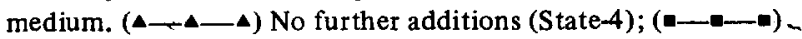
+ hexokinase, 1.3 units/ml (State-3); (•- $-\bullet)+$ hexokinase (1.3 units $/ \mathrm{ml})+1 \mu \mathrm{M} \mathrm{CCCP}$.

state of the mitochondrial AdN. It should be noted however, that the in vivo regulation of pyruvate carboxylation is still a matter of debate [cf. 9,10].

\subsection{Ketogenesis: Comparison of pyruvate and fatty acids as precursors}

It follows from the preceding section that the concentration of Krebs-cycle intermediates is an important factor in the control of ketogenesis from pyruvate, whereas the energy state per se has only a minor influence. On the other hand, in the case of ketogenesis from fatty acids both the concentration of Krebs-cycle intermediates and the energy state are important parameters in the control of the acetyl-ratio $[27,30]$. This discrepancy between fatty-acid and pyruvate oxidation may be explained by the observation that during pyruvate oxidation in State-4 the HB/Acac ratio is lower than unity (table 1 ), where as during palmitylcarnitine oxidation this ratio may reach values of five or higher [27]. An increase of the mitochondrial $\mathrm{NADH} / \mathrm{NAD}^{+}$ratio lowers the level of oxaloacetate, the reby limiting the activity of citrate syn thase and stimulating ketogenesis [31; cf. 32].

The lower HB/Acac ratios observed during pyruvate oxidation as compared with palmitylcarnitine oxidation may be caused by several factors: i) A continuous consumption of ATP in the carboxylation of pyruvate; ii) A low capacity of pyruvate dehydrogenase to produce $\mathrm{AcCoA}$ and reducing equivalents as compared with the $\beta$-oxidation of fatty acids; and iii) Consumption of NADH in the formation of malate from oxaloace tate following pyruvate carboxylation.

In short, we propose that a State- 3 to State- 4 transition lowers ketogenesis from pyruvate by activating pyruvate carboxylase. The newly formed oxaloace tate diverts $\mathrm{AcCOA}$ from acetoacetate synthesis to the formation of citrate. On the other hand, in the case of fatty-acid oxidation ketogenesis is stimulated when going from a low to a high energy state. This stimulation reflects the decreased intramitochondrial oxaloace tate concentration caused by the drastically elevated $\mathrm{NADH} / \mathrm{NAD}^{+}$ratio during fatty-acid oxidation in State-4.

\subsection{Effects of fatty acid oxidation and palmityl-CoA on pyruvate metabolism}

Upon addition of increasing amounts of palmityl$\mathrm{CoA}$ to mitochondria oxidizing pyruvate under State-3 conditions, parallel increases in the ratio of pyruvate carboxylation over pyruvate decarboxylation and the $\mathrm{HB} /$ Acac ratio are observed (table 2). Either a suboptimal amount of atractylate or the omission of hexokinase have analogous effects. The inhibition of mitochondrial AdN transport by palmityl-CoA leads to an increase of the mitochondrial phosphate potential, which in turn enhances the rate of pyruvate carboxylation. This stimulation of pyruvate carboxylation by palmityl-CoA is still more striking in the presence of 
Table 2

Effects of added palmityl-CoA on pyruvate metabolism.

\begin{tabular}{|c|c|c|c|c|c|}
\hline \multirow{3}{*}{ Additions } & \multirow{3}{*}{$\begin{array}{l}\begin{array}{l}\text { Oxygen } \\
\text { uptake }\end{array} \\
\text { (umoles) }\end{array}$} & \multicolumn{3}{|c|}{ Pyruvate } & \multirow{3}{*}{$\frac{\mathrm{HB}}{\mathrm{Acac}}$} \\
\hline & & Used & Carboxylated & \multirow{2}{*}{$\frac{\text { Carboxylated }}{\text { Decarboxylated }}$} & \\
\hline & & \multicolumn{2}{|c|}{ (umoles) } & & \\
\hline None & 3.7 & 3.00 & 0.22 & 0.08 & 0.05 \\
\hline PCoA (2 nmoles/mg) & 3.6 & 3.39 & 0.34 & 0.11 & 0.13 \\
\hline PCoA (5 nmoles/mg) & 4.2 & 3.74 & 0.60 & 0.19 & 0.17 \\
\hline PCoA (10 nmoles $/ \mathrm{mg})$ & 3.2 & 4.07 & 1.31 & 0.47 & 0.37 \\
\hline Atractylate & 4.1 & 3.67 & 0.59 & 0.19 & 0.15 \\
\hline Nonet & 2.5 & 4.67 & 2.37 & 1.03 & 0.44 \\
\hline Palmitate & 5.6 & 2.72 & 0.38 & 0.16 & 0.08 \\
\hline \multicolumn{6}{|l|}{ Palmitate + PCoA } \\
\hline (5 nmoles/mg) & 5.9 & 3.54 & 1.47 & 0.71 & 0.26 \\
\hline
\end{tabular}

$\dagger$ Hexokinase was omitted in this experiment.

To the standard reaction medium 5.2 units of hexokinase and $3 \mathrm{mM}$ pyruvate were added. Further additions: palmityl-CoA (PCoA) as indicated, $2.0 \mu \mathrm{g}$ atractylate, $0.5 \mathrm{mM}$ palmitate. Mitochondrial protein, $10.3 \mathrm{mg}$.

Table 3

Stimulation of pyruvate carboxylation by palmityl-CoA generated from palmitylcarnitine.

\begin{tabular}{|c|c|c|c|c|}
\hline \multirow{3}{*}{ Additions } & \multicolumn{3}{|c|}{ Pyruvate } & \multirow{3}{*}{$\frac{\mathrm{HB}}{\mathrm{Acac}}$} \\
\hline & Used & Carboxylated & \multirow{2}{*}{$\frac{\text { Carboxylated }}{\text { Decarboxylated }}$} & \\
\hline & \multicolumn{2}{|c|}{ (umoles) } & & \\
\hline None & 3.89 & 0.21 & 0.06 & 0.10 \\
\hline Palmitate & 3.46 & 0.32 & 0.10 & 0.07 \\
\hline CoASH $(100 \mu \mathrm{M})$ & 3.91 & 0.33 & 0.09 & 0.11 \\
\hline Palmitate + CoASH $(100 \mu \mathrm{M})$ & 3.65 & 1.17 & 0.47 & 0.51 \\
\hline Palmitate + CoASH $(100 \mu M)+C n$ & 3.74 & 0.47 & 0.14 & 0.10 \\
\hline $\mathrm{PCn}$ & 2.46 & 0.94 & 0.62 & 0.16 \\
\hline $\mathrm{PCn}+\mathrm{CoASH}(20 \mu \mathrm{M})$ & 2.62 & 1.34 & 1.05 & 0.36 \\
\hline
\end{tabular}

The standard reaction medium was supplemented with 2.6 units of hexokinase and 3 mM pyruvate. Further additions: $0.1 \mathrm{mM}$ palmitate, CoASH as indicated, $0.5 \mathrm{mM} \mathrm{L-carnitine} \mathrm{(Cn),} 0.5 \mathrm{mM}$ L-palmitylcarnitine (PCn). Mitochondrial protein, $11.3 \mathrm{mg}$.

an additional amount of palmitate. In view of the observed increase in oxygen uptake, this effect of added palmitate can be explained by the stimulation of oxidative phosphorylation as a consequence of palmitate oxidation. This will elevate the mitochondrial ATP/ADP ratio even further. A similar effect of oleyl-CoA was reported by Stucki et al. [17].
In the experiments shown in table 3, palmityl-CoA was generated during the incubations from either palmitate + CoASH or L-palmitylcarnitine + $\mathrm{CoASH}$ [25]. In the former case palmityl-CoA is formed by the palmityl-CoA synthetase localized in the outer mitochondrial membrane [33], in the latter case by carnitine palmityltransferase [34]. In both cases ex tra- 
mitochondrial palmityl-CoA accumulates due to the impermeability of the inner mitochondrial membrane towards acyl-CoA and CoASH [35]. The results in table 3 are entirely consistent with the effects of added palmityl-CoA (table 2) and they also show that L-carnitine counteracts these effects. Parenthetically, it may be noted that palmitate (table 2 ) as well as palmitylcarnitine (table 3 ) lower the rate of pyruvate decarboxylation in agrecment with the wcll-documented sparing effect of fatty acids on pyruvate oxidation.

Halperin et al. [36] recently reported that palmitylCoA has an inhibitory effect on the translocation of citrate and malate in rat liver mitochondria. However, in our experiments no interference with the accumulation of malate, fumarate and citrate (the main products of pyruvate carboxylation) could be detected, showing that in our system the $\mathrm{AdN}$ translocation rather than the transport of these Krebs-cycle intermediates is rate-limiting.

In conclusion, the following effects occur when palmitate is added to mitochondria oxidizing pyruvate: i) An acceleration of ATP synthesis; and ii) Accumulation of palmityl-CoA slowing down the exchange of AdN through the inner mitochondrial membrane. Both effects will cause an increase in the intramitochondrial ATP/ADP ratio and a concomitant stimulation of pyruvate carboxylation. In addition, the mitochondrial pool of exchangeable nucleotides will be lowered by an increased AMP level due to intramitochondrial palmitate activation [17]. A regulatory role of the $\mathrm{AcCoA} / \mathrm{CoA}$ ratio [cf. 8-11] remains to be established.

The present investigation points to a possible role of long-chain acyl-CoA esters in the regulation of gluconeogenesis in rat liver. Arguments in favour of this hypo.. thesis $[37,25]$ may be briefly summarized as follows: i) The increased levels of long-chain acyl-CoA observed in vivo during starvation $[37,38]$ simultaneously with a stimulated gluconeogenesis and ketogenesis; ii) The competitive inhibition of the AdN translocator by low levels of long-chain acyl-CoA and its reversal by albumin and L-carnitine [18-23]; iii) The increase in the mitochondrial phosphate potential [37] and the decrease of the cytosolic one [39] calculated to occur in starvation and in starvation followed by refeeding fat; and iv) The stimulation by palmityl-CoA of ketogenesis during palmitate oxidation [25] and of pyruvate carboxylation (present results) in isolated rat-liver mitochondria.

\section{Acknowledgements}

The skilful technical assistance of Miss W. Klazinga and Miss T.A. Wisserhof is gratefully acknowledged. This investigation was supported in part by the Netherlands Foundation for Chemical Research (S.O.N.) with financial aid from the Netherlands Organization for the Advancement of Pure Research (Z.W.O.).

\section{References}

[1] J.M. Lowenstein, in: The Metabolic Roles of Citrate, ed. T.W. Goodwin (Academic Press, London, 1968) p. 61.

[2] G.D. Greville, in: The Citric Acid Cycle, ed. J.M. Lowenstein (Dekker, New York, 1969) p. 1.

[3] J. Tepperman and H.M. Tepperman, Federation Proc. 29 (1970) 1284.

[4] L.J. Reed, in: Current Topics in Cellular Regulation, Vol. 1, eds. B.L. Horecker and E.R. Stadtman (Academic Press, New York, 1969) p. 233.

[5] J. Bremer, European J. Biochem. 8 (1969) 535.

[6] J.D. Erfle and F. Sauer, Biochim. Biophys. Acta 178 (1969) 441.

[7] O. Wieland and E. Siess, Proc. Natl. Acad. Sci. U.S. 65 (1970) 947.

[8] M,F. Utter and M.C. Scrutton, in: Current Topics in Cellular Regulation, Vol. 1, eds. B.L. Horecker and E.R. Stadtman (Academic Press, New York, 1969) p. 253.

[9] W.R. McLure and H.A. Lardy, J. Biol. Chem. 246 (1971) 3591.

[10] M.C. Scrutton and M.D. White, Biochem. Biophys. Res. Commun. 48 (1972) 85.

[11] C.D. Seufert, E. Herlemann, E. Albrecht and W. Seubert, in: Regulation of Gluconeogenesis, eds. H.D. Söling and B. Willms (Thieme Verlag, Stuttgart, 1971) p. 11.

[12] J.M. Lowenstein, in: Comprehensive Biochemistry, Vol. $18 \mathrm{~S}$, eds. M. Florkin and E.H. Stotz (Elsevier, Amsterdam, $1971)$ p. 1.

[13] J. Bremer, Biochim. Biophys. Acta 116 (1966) 1 .

[14] D.G. Nicholls, D. Shepherd and P.B. Garland, Biochem. J. 103 (1967) 677.

[15] G. von Jagow, B. Westermann and O. Wieland, European J. Biochem. 3 (1968) 512.

[16] P. Walter and J.W. Stucki European J. Biochem. 12 (1970) 508.

[17] J.W. Stucki, F. Brawand and P. Walter, European J. Biochem. 27 (1972) 181.

[18] S.V. Pande and M.C. Blanchaer, J. Biol. Chem. 246 (1971) 402.

[19] W.J. Vaartjes and S.G. van den Bergh, Abstr. Commun. Meet. Fed. Eur. Biochem. Soc. 7 (1971) 227.

[20] W.J. Vaartjes, A. Kemp Jr., J.H.M. Souverijn and S.G. van den Bergh, FEBS Letters 23 (1972) 303. 
[21] A.L. Shug, E. Lerner, C. Elson and E. Shrago, Biochem. Biophys. Res. Commun. 43 (1971) 557.

[22] E. Lerner, A.L. Shug, C. Elson and E. Shrago, J. Biol. Chem. 247 (1972) 1513.

[23] R.A. Harris, B. Farmer and T. Ozawa, Arch. Biochem. Biophys. 150 (1972) 199.

[24] E. Pfaff, H.W. Heldt and M. Klingenberg, European J. Biochem. 10 (1969) 484.

[25] W.J. Vaartjes, M. Lopes-Cardozo and S.G. van den Bergh, FEBS Letters 26 (1972) 117.

[26] P. Walter, V. Paetkau and H.A. Lardy, J. Biol. Chem. 241 (1966) 2523.

[27] M. Lopes-Cardozo and S.G. van den Bergh, Biochim. Biophys. Acta 283 (1972) 1.

[28] D.H. Williamson, P. Lund and H.A. Krebs, Biochem. J. 103 (1967) 514.

[29] J. Bremer, Biochem. Prep. 12 (1968) 69.

[30] S.G. van den Bergh and M. Lopes-Cardozo, in: Energy Transduction in Respiration and Photosynthesis, eds. E. Quagliariello, S. Papa and C.S. Rossi (Adriatica Editrice, Bari, 1971) p. 421.
[31] M.S. Olson and J.R. Williamson, J. Biol. Chem. 246 (1971) 7794.

[32] P.B. Garland, D. Shepherd, D.G. Nicholls, D.W. Yates and P.A. Light, in: The Citric Acid Cycle, ed. J.M. Lowenstein (Dekker, New York, 1969) p. 163.

[33] A. van Tol and W.C. Hülsmann, Biochim. Biophys. Acta 223 (1970) 416.

[34] K.R. Norum, Biochim. Biophys. Acta 89 (1964) 95.

[35] I.B. Fritz and K.T.N. Yue, J. Lipid Res. 4 (1963) 279.

[36] M.L. Halperin, B.H. Robinson and I.B. Fritz, Proc. Natl. Acad. Sci. U.S. 69 (1972) 1003.

[37] P. McLean, K.A. Gumaa and A.L. Greenbaum, FEBS Letters 17 (1971) 345.

[38] P.K. Tubbs and P.B. Garland, Biochem. J. 93 (1964) 550.

39] R.L. Veech, L. Raijman and H.A. Krebs, Biochem. J. 117 (1970) 499. 